\title{
Aspectos psicopatológicos e forenses de mulheres homicidas com diagnóstico de transtornos psicóticos primários - estudo de série de casos
}

\author{
Psychopatologic and forensic features of female murderes with \\ a diagnosis of primary psycchotic disorders - a case series
}

Alexandre Martins Valença' ${ }^{\top}$

\section{RESUMO}

Objetivo: Avaliar amostra de todas as mulheres com diagnóstico de transtorno psicótico primário que receberam medida de segurança e estavam em hospital de custódia e tratamento psiquiátrico em regime de internação $(n=8)$, em virtude de homicídio ou tentativa de homicídio (artigo 121 do Código Penal Brasileiro). Métodos: Trata-se de um estudo de série de casos. Foi realizado o levantamento retrospectivo de dados por meio de registros periciais e pareceres de equipes de assistência. O diagnóstico psiquiátrico final foi realizado com base na entrevista psiquiátrica e observação dos registros, utilizando-se os critérios diagnósticos do DSM-IV-TR. Houve aplicação de questionário padronizado e escala das sín-

\section{Palavras-chave}

Homicídio, sexo feminino, transtorno mental, medida de segurança. dromes positiva e negativa (PANSS). Resultados: Sete pacientes receberam diagnóstico de esquizofrenia e uma de transtorno esquizoafetivo. A maioria das vítimas $(n=6,60 \%)$ era membro familiar das pacientes. Foi encontrado que $37,5 \%(n=3)$ das pacientes tinham história prévia de comportamento violento. De acordo com a avaliação da perícia psiquiátrica inicial, cinco (62,5\%) pacientes da amostra total apresentavam sintomatologia psicótica no momento desta avaliação. As alucinações auditivas foram os sintomas psicóticos mais comuns. Conclusão: $\mathrm{O}$ estudo de fatores motivadores do comportamento homicida pode fornecer conhecimentos para o estabelecimento de intervenções terapêuticas em mulheres com transtornos mentais que apresentem risco para este ou outros comportamentos violentos.

\begin{abstract}
Objective: Evaluate a sample of women with primary psychotic disorders who received a safety measure and were admitted to inpatient forensic psychiatric care $(n=8)$, due to homicide or attempted homicide (article 121 from the Brazilian Penal Code). Method: This is a serial cases study. The case records of all the women were retrospectively examined, including technical files and the evaluations of clinical experts. The final psychiatric diagnosis was obtained with a psychiatric evaluation of the patients and the examination of their records, using the diagnostic criteria of DSM-IV-TR. A standardized interview and positive and negative syndrome scale (PANSS) were
\end{abstract}

Psiquiatra Forense pela Associação Brasileira de Psiquiatria. 1 Departamento de Psiquiatria e Saúde Mental, Centro de Ciências Médicas da Universidade Federal Fluminense (UFF) e Programa de Pósgraduação em Psiquiatria e Saúde Mental do Instituto de Psiquiatria da Universidade Federal do Rio de Janeiro (UFRJ). 


\section{Keywords}

Homicide, female gender, mental disorder, safety measure. also applied. Results: Seven patients received a diagnosis of schizophrenia and one a diagnosis of schizoaffective disorder. Most victims $(n=10 ; 60 \%)$ were family members of the patients. We found that $37.5 \%(n=3)$ of the patients had previous history of violent behavior. According to the initial psychiatric forensic evaluation, five (62.5\%) of the patients from the sample had psychotic symptoms. Auditory hallucinations were the most common psychotic symptoms. Conclusion: The study of motivational factors for homicidal behavior may provide further knowledge for the implantation of therapeutic interventions in women with mental disorders and an increased risk for homicidal or other violent behaviors.
O homicídio é um dos crimes mais antigos da humanidade. Está incurso no artigo 121 da parte especial do Código Penal Brasileiro de 1984' que é: "Matar alguém". A Lei no 8.072/1990, que dispõe sobre crimes hediondos, após a nova redação introduzida pela Lei no 8.930/1994, incluiu o homicídio como crime hediondo. Isso representou tentativa de conter a criminalidade, impondo resposta punitiva mais severa para os homicidas 2 . O cumprimento da pena, para os crimes considerados hediondos, deve ser feito em regime integralmente fechado, impedindo, assim, a progressão para fases mais leves de execução (regime semi-aberto e aberto). A pena para o homicídio simples pode ser de seis a 20 anos de reclusão; já para o homicídio qualificado, a pena pode variar entre 12 a 30 anos, instituída pelo Código Penal Brasileiro.

A violência cometida por indivíduos com transtornos mentais graves tem se tornado crescente foco de interesse entre médicos, autoridades policiais e população em geral. Diversos estudos, na última década, têm mostrado associação entre transtornos mentais e comportamento violento3,4. Uma das principais abordagens para estudar esta relação são as pesquisas com indivíduos homicidas, uma vez que o homicídio é considerado expressão mais grave da violência.

Quando se estuda a relação entre homicídio e transtornos mentais, um conceito importante é o de homicídio "anormal". Este é assim denominado por sua bizarria e incompreensibilidade 5 , sendo também reconhecido pelo exame psiquiátrico do perpetrador e sua caracterização como indivíduo que apresenta transtorno mental grave. A incidência de homicídios anormais tende a ser constante em diversas sociedades, acompanhando a incidência dos principais transtornos mentais, enquanto a incidência de homicídios "normais" (em que não há presença de doença mental) reflete realidades mais amplas de uma sociedade, como a violência urbana, por exemplo.

Até o início dos anos 1980, havia consenso de que a esquizofrenia não levaria a risco maior de comportamento violento do que aquele encontrado na população geral. Entretanto, novas evidências epidemiológicas têm se acumulado nos últimos 20 anos, indicando que indivíduos com transtornos mentais graves, especialmente aqueles com esquizofrenia, apresentam risco maior (comparados à popula- ção geral) de cometer crime violento, embora a proporção de violência social atribuível a este risco seja pequena ${ }^{6}$. Para indivíduos com transtornos psicóticos, considera-se que a própria condição psicopatológica seja fator de risco para comportamento violento.

A associação entre esquizofrenia e comportamento violento é achado robusto: tem sido descrito por vários grupos de pesquisadores independentes que trabalham em países industrializados ${ }^{4}$ e em países em desenvolvimento? com diferentes culturas, serviços sociais e de saúde e sistemas de justiça criminal, em estudos examinando diferentes amostras e utilizando metodologias diversas. Estes achados implicam grande sofrimento, tanto para as vítimas quanto para os perpetradores, além de custo financeiro alto para a sociedade. Além disso, evidências têm encontrado que pessoas com transtornos mentais graves têm risco maior de serem vítimas de agressão física ${ }^{8}$.

Um dado curioso é que há menos estudos de homicídios anormais em amostras formadas apenas por mulheres, comparados aos estudos com homens. Embora diversos estudos mostrem associação significativa entre transtornos mentais graves e violência, ainda não está estabelecido porque alguns pacientes apresentam comportamento violento e outros não. Para Hodgins ${ }^{6}$, o comportamento violento está associado a fatores contextuais e individuais. Entre os primeiros estão incluídos repressão policial e disponibilidade e adequação de tratamento psiquiátrico. Entre os segundos estão a presença de transtornos de personalidade comórbidos e transtornos relacionados ao uso de álcool e/ou drogas, o que é reforçado por outros autores ${ }^{9}$, além de falta de aderência ao tratamento e ausência de insight em relação ao transtorno ${ }^{10}$. De acordo com Nestor ${ }^{11}$, pessoas que apresentam abuso de substâncias têm risco de 12 a 16 vezes maior de se envolver em comportamento violento do que outras que não usam substâncias.

Constatou-se que na literatura há escassez de estudos sobre a relação entre comportamento violento e transtornos mentais nos países em desenvolvimento, especialmente de estudos que pesquisem populações de mulheres.

O objetivo desse estudo é avaliar, do ponto de vista psiquiátrico-forense, uma população de mulheres com 
diagnóstico de transtornos psicóticos primários, internadas e cumprindo medida de segurança em um Hospital de Custódia e Tratamento Psiquiátrico do estado do Rio de Janeiro, por delito de homicídio ou tentativa de homicídio.

\section{HOMICÍDIO E TRANSTORNOS PSICÓTICOS}

Vários estudos têm investigado grupos específicos de pacientes com transtornos mentais, com a finalidade de estabelecer correlações entre comportamento violento ou homicida e variáveis sociodemográficas e psicopatológicas. Sem dúvida, a esquizofrenia é o transtorno mental mais bem estudado neste aspecto.

Wallace et al.12 examinaram os antecedentes criminais de 2.861 indivíduos que tiveram a primeira internação hospitalar por causa da esquizofrenia, dos anos de 1975 a 2000, sendo comparados a igual número de indivíduos na comunidade, pareados por idade, sexo e região residencial. Foram considerados atos violentos: agressão, lesão física séria e homicídio. Observou-se que os pacientes com esquizofrenia foram significativamente mais condenados por, pelo menos, um ato violento, comparados aos indivíduos da comunidade ( $8,2 \%$ e $1,8 \%$, respectivamente). Outro achado importante é que os pacientes com problemas de abuso de substâncias tiveram mais condenações por delitos do que aqueles que não utilizavam drogas (68\% e $11,7 \%$, respectivamente). Comparados ao grupo controle, indivíduos com esquizofrenia tiveram risco 3,6 a 6,6 vezes maior de ter, pelo menos, uma condenação por comportamento violento.

Swanson et al. ${ }^{13}$ realizaram estudo com dados obtidos por meio do projeto Catie, que foi o ensaio randomizado conduzido entre os anos de 2001 e 2004, em 57 centros clínicos de 24 estados dos Estados Unidos, contando com 1.460 indivíduos esquizofrênicos. Foi considerado como violência menor a agressão sem uso de arma de fogo ou produção de lesão na vítima. Já a agressão letal ou produtora de lesão grave, uso de arma de fogo e qualquer forma de violência sexual foi considerada como violência maior. Dos 1.410 participantes deste estudo, 1.140 (81\%) não relataram comportamento violento, 219 (15,5\%) relataram violência menor e 51 (3,6\%) relataram violência grave. Foi encontrado que a violência menor foi significativamente maior entre os participantes com muitas características não clínicas: idade jovem, sexo feminino, atividade vocacional inexistente ou limitada, residindo com familiares, não se sentindo "escutado" por membros familiares e histórico recente de contato policial.

Ainda em relação ao estudo de Swanson et al!', no domínio clínico, a violência menor foi associada com sintomas psicóticos positivos (escores de sintomas positivos mais elevados na escala PANSS ${ }^{14}$ ). Já idade jovem, problemas de conduta na infância e história de prisão foram significativamente associados com violência grave. Entre as variáveis clínicas, escores acima da média de sintomas positivos aumentaram o risco de violência grave, porém escores acima da média de sintomas negativos diminuíram esse risco. $\mathrm{O}$ abuso/dependência de substâncias foi associado a aumento de quatro vezes do comportamento violento grave. Entre os sete sintomas que compõem a subescala positiva do PANSS ${ }^{14}$, cinco sintomas específicos (hostilidade, suspicácia/ perseguição, comportamento alucinatório, grandiosidade e excitação) foram associados significativamente com maior risco de violência grave.

Em outro estudo sobre comportamento violento na esquizofrenia, Meehan et al.15 utilizaram uma pesquisa clínica nacional que coletou dados de pessoas condenadas por homicídio na Inglaterra, entre os anos de 1996 a 1999. De 1.594 indivíduos acusados de homicídio, 85 (5\%) apresentavam esquizofrenia. A maioria era homens (86\%). Um terço (32\%) tinha sido previamente condenada por violência. Vinte e quatro perpetradores (28\%) nunca tinham tido contato com serviços de saúde mental e 18 perpetradores tinham tido este contato, porquanto o mesmo não ocorreu no ano anterior à ofensa. Apesar de muitos deles estarem doentes há muitos anos, houve evidência de deterioração clínica recente, incluindo mudança e intensificação dos delírios antes da ofensa criminal, daí a importância de monitoração e avaliação dos delírios e mudança na intensidade deles, em indivíduos com esquizofrenia.

Volavka et al.7, em um estudo transcultural, fundamentado em informações de 1.017 pacientes esquizofrênicos, verificou que 209 (20,6\%), tinham história de agressão, que foi mais comum nos países em desenvolvimento $(31,5 \%)$ do que nos países desenvolvidos (10,5\%) e nos pacientes mais jovens e de baixo status socioeconômicos. Na maioria dos casos (57,7\%), a agressão coincidiu com o início do transtorno esquizofrênico. Houve aumento significativo do risco de agressão em pacientes com problemas sérios de uso de álcool, comparados àqueles com problemas leves ou sem problemas relacionados a esta substância.

Continuando a estudar o mesmo tema, Nielssen et al. ${ }^{16}$ descreveram homicídios praticados por indivíduos com transtornos psicóticos no Reino Unido, em um período de dez anos (1993-2002). A amostra do estudo foi composta por uma série de homens e mulheres que mataram durante episódio psicótico e foram considerados inocentes em virtude de insanidade mental ou tiveram a defesa de alegação de doença mental disponível em cortes judiciais, após avaliação psiquiátrica. Neste período ocorreram 1.052 homicídios, sendo 93 (8,8\%) praticados durante transtorno psicótico, por 88 indivíduos com este transtorno. Uma elevada proporção de indivíduos da amostra relatou abuso de substâncias: 57 homens (80\%) e sete mulheres (41\%). As substâncias mais utilizadas foram cannabis sativa (59\%), anfetamina 
(26\%) e álcool (23\%). Trinta e um indivíduos (35\%) relataram intoxicação no momento do homicídio.

Um estudo epidemiológico de Link e Stueve ${ }^{17}$ encontrou que subgrupos particulares de delírios com características de controle e perseguição aumentavam fortemente o risco de comportamento violento. Estes achados foram reforçados pelo estudo de Beck ${ }^{18}$ que encontrou relação positiva entre delírio, abuso de substância e crime violento. Cheung et al. ${ }^{19}$ compararam 31 pacientes esquizofrênicos com outros 31 não-violentos, todos de enfermaria psiquiátrica comum e sem comorbidade com uso de substâncias psicoativas. Foi encontrado que os pacientes do grupo violento eram mais influenciados por delírios persecutórios, enquanto os do grupo não-violento eram mais influenciados por delírios de grandeza.

\section{MÉTODOS}

No Brasil, o sistema penal, por imperativo legal, transfere os criminosos doentes mentais para hospitais de custódia ou para o hospital psiquiátrico penitenciário. No estado do Rio de Janeiro, existem três instituições psiquiátricas que fazem parte do Departamento de Sistema Penitenciário. A partir do ano de 2006, o Hospital de Custódia e Tratamento Psiquiátrico Heitor Carrilho tornou-se o único hospital de custódia para cumprimento de medida de segurança de muIheres, em regime de internação hospitalar, no estado do Rio de Janeiro. Indivíduos do sexo masculino também são internados neste hospital. No momento da presente pesquisa, 31 mulheres estavam cumprindo medida de segurança, por determinação judicial. Foram selecionadas para esta pesquisa todas as pacientes com diagnóstico de transtorno psicótico primário que estavam cumprindo medida de segurança por infração ao artigo 121 do Código Penal do Brasil (homicídio ou tentativa de homicídio) $(n=8)$. Trata-se de um estudo de série de casos. Os transtornos psicóticos primários foram definidos como esquizofrenia, transtorno esquizoafetivo e transtorno delirante.

Todas as pacientes participantes do estudo foram avaliadas previamente em perícia psiquiátrico-forense (laudo de exame de sanidade mental). Também foram examinadas pelo autor do presente estudo, entre os meses de julho e dezembro de 2007. Os dados de identificação se referem à época do delito, com a finalidade de identificar variáveis sociodemográficas na ocasião deste. Foi realizado levantamento de dados de registros periciais e pareceres de equipe de assistência do manicômio judiciário, em que as examinadas se encontravam internadas. O diagnóstico psiquiátrico final foi estabelecido com base na entrevista psiquiátrica e observação dos registros, utilizando-se os critérios diagnósticos do Manual Diagnóstico e Estatístico dos Transtornos Mentais (4a edição, texto revisado) ${ }^{20}$.
A primeira etapa da investigação consistiu do levantamento de informações colaterais (história criminal, circunstâncias dos crimes atuais, outras informações relevantes) por meio dos laudos periciais e prontuários individuais, bem como do exame psicopatológico dos indivíduos. Foram utilizados os seguintes instrumentos para a realização da pesquisa: entrevista psiquiátrica utilizando os critérios do DSMIV-TR ${ }^{20}$ para estabelecimento do diagnóstico psiquiátrico; questionários padronizados sobre dados sociodemográficos e clínicos das pacientes; e escala das síndromes positiva e negativa (PANSS), no grupo de pacientes que apresentaram diagnóstico de transtornos psicóticos primários $(n=8)$.

Após concordância da paciente em participar da pesquisa, houve aplicação de entrevistas estruturadas e questionários pelo pesquisador do presente projeto. Não houve nenhuma interferência em relação ao tratamento recebido pelas pacientes, por parte das equipes de assistência da instituição mencionada.

As pacientes assinaram o termo de consentimento livre e esclarecido para participar voluntariamente do estudo. O estudo foi aprovado por parte do Comitê de Ética e Pesquisa local.

\section{RESULTADOS}

A amostra foi composta por oito pacientes do sexo feminino, acusadas de homicídio $(n=5 ; 62,5 \%)$ ou tentativa de homicídio ( $n=3 ; 37,5 \%$ ). Todas as pacientes aceitaram participar do estudo. Os dados sociodemográficos da amostra estão descritos na Tabela 1. Sete pacientes receberam diagnóstico de esquizofrenia, e uma de transtorno esquizoafetivo. Outras variáveis clínicas, como acompanhamento psiquiátrico, uso de psicofármacos ou uso de álcool/substâncias psicoativas previamente ao delito, estão descritas na Tabela 2.

No total houve dez vítimas agredidas, já que uma das pacientes tentou afogar seus três filhos menores de idade. A maioria das vítimas $(n=6,60 \%)$ era membro familiar das pacientes. Os principais instrumentos utilizados para a prática do delito foram os cortantes $(n=3,37,5 \%)$. Foi encontrado que 37,5\% ( $n=3)$ das pacientes tinham história prévia de comportamento violento. No que diz respeito ao grau de imputabilidade relacionado ao delito, a maioria das pacientes ( $n=7,87,5 \%)$ foi considerada inimputável (Tabela 3).

De acordo com a avaliação da perícia psiquiátrica inicial, cinco $(62,5 \%)$ pacientes da amostra $(n=8)$ apresentavam sintomatologia psicótica no momento desta avaliação. Observou-se que as alucinações auditivas $(n=4,50 \%)$ foram os sintomas psicóticos mais comuns neste grupo, ainda conforme a avaliação pericial. Quando as próprias pacientes deste grupo ( $n=8)$ foram questionadas sobre a existência de sintomatologia psicótica à época do delito, quatro (50\%) referiram alucinações auditivas (Tabela 3). 
Tabela 1. Dados sociodemográficos da amostra $(n=8)$.

\begin{tabular}{lc}
\hline Idade à época do delito (média \pm DP) & $35,6 \pm 11,5$ anos \\
Idade atual (média \pm DP) & $45,6 \pm 7,24$ anos \\
Cor & \\
Branca & $3(37,5 \%)$ \\
Preta & $4(50 \%)$ \\
Parda & $1(12,5 \%)$ \\
Naturalidade & $2(25 \%)$ \\
Rio de Janeiro & $6(75 \%)$ \\
Outros estados & $4,1 \pm 3,8$ anos \\
Escolaridade (anos) (média \pm DP) & \\
Estado civilà época do delito & $7(87,5 \%)$ \\
Solteira & $1(12,5 \%)$ \\
Casada/Amasiada & \\
Atividade profissional à época do delito & $3(37,5 \%)$ \\
Nunca trabalhou & $4(50 \%)$ \\
Informal & $1(12,5 \%)$ \\
Desempregado & \\
Religiäo & \\
Católica & $5(62,5 \%)$ \\
Evangélica & $2(25 \%)$ \\
Nenhuma & $1(12,5 \%)$ \\
Renda familiar & \\
Até 1 salário mínimo & \\
1 a 3 salários mínimos & $4(50 \%)$ \\
Mais de 5 salários mínimos & $2(25 \%)$ \\
Não sabe informar & $1(12,5 \%)$ \\
Residindo à época do delito & $1(12,5 \%)$ \\
Com familiares & \\
Com cônjuge/companheiro & $5(62,5 \%)$ \\
Sozinho & $1(12,5 \%)$ \\
Outros & $1(12,5 \%)$ \\
\hline & $1(12,5 \%)$ \\
\hline
\end{tabular}

Tabela 2. Variáveis clínicas da amostra $(n=8)$.

\begin{tabular}{lc}
\hline Diagnóstico (DSM IV-TR) & \\
Esquizofrenia paranóide & $4(50 \%)$ \\
Esquizofrenia residual & $3(37,5 \%)$ \\
Transtorno esquizoafetivo & $1(12,5 \%)$ \\
História prévia de uso de álcool/substância & \\
Sim & $3(37,5 \%)$ \\
Não & $5(62,5 \%)$ \\
Uso de álcool/substância nos dias anteriores ao delito & \\
Sim & $3(37,5 \%)$ \\
Não & $5(62,5 \%)$ \\
História prévia de tratamento psiquiátrico & \\
Sim & $6(75 \%)$ \\
Não & $2(25 \%)$ \\
História de tratamento psiquiátrico pouco antes ao delito & \\
Sim & $2(25 \%)$ \\
Não & $6(75 \%)$ \\
Uso de medicação psicotrópica pouco antes do delito & \\
Sim & $2(25 \%)$ \\
Não & $6(75 \%)$ \\
Número de processos criminais & \\
Um & $6(75 \%)$ \\
Dois & $1(12,5 \%)$ \\
Cinco & $1(12,5 \%)$ \\
\hline
\end{tabular}

Ainda em relação ao grupo estudado, a média e o desvio-padrão em relação aos escores de sintomas positivos e negativos medidos por meio da escala PANSS foi de $12 \pm$ 8,92 e $38,5 \pm 10,91$, respectivamente.
Tabela 3. Variáveis forenses da amostra $(n=8)$.

\begin{tabular}{lc}
\hline Classificação das vítimas & \\
Da família" & $6(60 \%)$ \\
Conhecida & $3(30 \%)$ \\
Desconhecida & $1(10 \%)$ \\
Meios/instrumentos usados no crime & \\
Atirar de altura elevada & $1(12,5 \%)$ \\
Afogamento & $1(12,5 \%)$ \\
Tortura ou privação alimentar & $1(12,5 \%)$ \\
Instrumento cortante & $3(37,5 \%)$ \\
Instrumento contundente & $1(12,5 \%)$ \\
Arma de fogo & $1(12,5 \%)$ \\
Comportamento violento prévio & \\
Sim & $3(37,5 \%)$ \\
Não & $5(62,5 \%)$ \\
Grau de inimputabilidade na avaliação pericial & \\
Semi-imputável & $1(12,5 \%)$ \\
Inimputável & $7(87,5 \%)$ \\
Sintomatologia psicótica na perícia inicial & \\
Sim & $5(62,5 \%)$ \\
Não & $3(37,5 \%)$ \\
Sintomas psicóticos de acordo com a avaliação pericial & \\
Ausente & $3(37,5 \%)$ \\
Delírios persecutórios & $2(25 \%)$ \\
Alucinaçōes auditivas & $4(50 \%)$ \\
Dissociação do pensamento & $1(12,5 \%)$ \\
\hline
\end{tabular}

"Uma paciente tentou matar seus três filhos menores de idade.

Em relação ao filicídio/tentativa de filicídio, três (37,5\%) das oito pacientes cometeram este delito. Ao todo cinco crianças foram agredidas, visto que uma veio a falecer. A idade das crianças variou de 1 a 7 anos. A criança morta tinha 1 ano de idade. Três crianças eram do sexo masculino e duas do sexo feminino. No que diz respeito ao método utilizado para agressão às crianças, três sofreram tentativa de afogamento, uma foi atingida com instrumento contundente na cabeça e outra foi atirada de andar alto. Esta última veio a falecer. Em todos os casos as pacientes eram as principais cuidadoras das crianças. Em apenas um caso havia história de abuso físico prévio à criança. Em dois casos havia história de abuso de álcool por parte da genitora.

\section{DISCUSSÃO}

No presente estudo foi realizada avaliação psiquiátrico-forense de amostra de oito pacientes com transtornos psicóticos primários que estavam cumprindo medida de segurança em hospital de custódia e tratamento psiquiátrico, por homicídio ( $n=5)$ ou tentativa de homicídio $(n=3)$, na cidade do Rio de Janeiro. A maior parte destas apresentava baixo nível de escolaridade, eram solteiras (87,5\%), não tinham atividade profissional (50\%) e tinham baixa renda familiar, à época do delito. Sete $(87,5 \%)$ pacientes receberam diagnóstico de esquizofrenia e uma (12,5\%) de transtorno esquizoafetivo. Os achados do presente estudo ilustram dados da literatura que têm apontado a esquizofrenia como importante trans- 
torno mental relacionado ao comportamento violento em países desenvolvidos ${ }^{4}$ e em países em desenvolvimento?. O estudo da média e do desvio-padrão de escores de sintomas positivos e negativos neste grupo, por meio da escala PANSS, mostrou pontuação mais baixa dos primeiros (12 \pm $8,92)$ em relação aos últimos $(38,5 \pm 10,91)$ achado possivelmente relacionado à evolução deste transtorno mental grave, havendo predomínio de sintomas negativos.

Uma história passada de violência tem sido consistentemente considerada como preditiva de violência subsequente, em diversas populações de pacientes ${ }^{21}$. Da amostra do presente estudo, três pacientes (37,5\%) tinham história prévia de comportamento violento, porquanto duas estavam envolvidas em dois processos criminais e outra em cinco.

A relação entre transtornos mentais graves e criminalidade é mais complexa do que simples causalidade. Fatores como idade, gênero, status socioeconômico e criminalidade prévia são importantes, assim como outros fatores potencialmente tratáveis, como abuso de substâncias, transtornos de personalidade e uso regular de medicamentos. A comorbidade com abuso de substâncias aumenta o risco de comportamento violento naqueles com transtornos mentais graves ${ }^{3,4}$. Neste aspecto, foram encontradas na amostra deste estudo que três de oito pacientes (37,5\%) usaram álcool nos dias anteriores ao delito e uma delas, além de álcool, também utilizou cannabis sativa e cocaína, previamente ao delito. Curiosamente, duas pacientes que tinham envolvimento em mais de um processo criminal tinham história de abuso de álcool. Uma delas, envolvida em cinco processos criminais, além de álcool também utilizava cannabis sativa e cocaína.

No presente estudo, cinco (62,5\%) pacientes da amostra total $(n=8)$ apresentavam sintomatologia psicótica no momento da avaliação pericial inicial (laudo de exame de sanidade mental). Verificou-se que as alucinações auditivas ( $n=4$ pacientes, $50 \%$ ) foram os sintomas psicóticos mais comuns neste grupo, ainda de acordo com a avaliação pericial. Quando as próprias pacientes deste grupo $(n=8)$ foram questionadas no momento do presente estudo sobre a existência de sintomatologia psicótica à época do delito, quatro (50\%) referiram alucinações auditivas. Certamente este último dado tem valor limitado e fidedignidade incerta, por depender da lembrança das pacientes. O presente estudo, também, ilustra a importância da sintomatologia psicótica antes da manifestação do comportamento violento.

Homicídio e agressões despropositadas são frequentemente resultados de sintomas que levaram pessoas com transtornos mentais a acreditar que estavam em perigo. Um estudo de Taylor ${ }^{22}$ encontrou forte associação entre sintomas psicóticos e comportamento violento recente, já que 93\% desta amostra apresentava sintomatologia psicótica quando cometeram estes crimes e $47 \%$ foram "definitivamente" ou "provavelmente" motivados por estes crimes.
Outros estudos têm encontrado associação entre alucinações auditivas e idéias delirantes persecutórias e motivação para homicídio ${ }^{18,19}$

Na amostra estudada, o comportamento violento foi mais dirigido contra membros familiares. Das dez vítimas de homicídio ou tentativa de homicídio, seis (60\%) eram familiares das pacientes, incluindo irmão e filhos. Uma paciente tentou afogar seus três filhos, que conseguiram sobreviver. É possível que o comportamento violento dirigido a membros familiares ocorra por causa da organização da convivência. Os homens podem passar mais tempo fora de casa, em contato com conhecidos e estranhos, já a mulher tende a permanecer mais tempo em ambiente doméstico, em contato com familiares ${ }^{23}$. Assim, é importante que os clínicos considerem o potencial de violência por homens e mulheres igualmente sério e importante. É preciso levar em conta diferentes fatores situacionais na predição de comportamento violento em ambos os sexos, e, dessa forma, planejar e realizar intervenções terapêuticas para todos os indivíduos com risco de manifestação deste comportamento.

Em relação ao filicídio/tentativa de filicídio, três pacientes $(37,5 \%)$ da amostra estudada cometeram este delito. Duas delas tinham histórico prévio de uso abusivo de derivados etílicos. Embora os estudos prospectivos sejam considerados mais fidedignos, do ponto de vista metodológico, para o estabelecimento de fatores de risco, a relativa raridade do filicídio torna difícil a realização destes. Estudos futuros devem investigar diversos fatores de risco para o filicídio, como depressão, transtornos psicóticos e fatores sociodemográficos, meio sociocultural, história psiquiátrica prévia, história de conflitos e violência familiar.

Para Friedman et al.24, psiquiatras, obstetras e pediatras deveriam fazer avaliação quanto a presença de sintomas psiquiátricos no período pós-parto. Mulheres que solicitam ajuda para depressão e psicose necessitam de resposta imediata dos sistemas de saúde mental. Mulheres deprimidas ou psicóticas deveriam ser questionadas se apresentam pensamentos ou medo de ferir suas crianças. Mulheres com delírios relacionados à criança, ideação suicida ou psicose deveriam ser avaliadas em relação ao risco de suicídio. A indicação de hospitalização psiquiátrica deveria ser avaliada nos casos de mulheres psicóticas ou gravemente deprimidas, que possam colocar suas crianças em risco.

Diversos estudos têm encontrado padrão duradouro de rompimento do contato com serviços de saúde mental, enquanto em outros o homicídio parece ocorrer logo após o início do transtorno mental, antes do ofensor ter estabelecido contato com estes serviços ${ }^{15}$. No presente estudo, observouse que seis de oito pacientes (75\%) tinham história prévia de tratamento psiquiátrico. Entretanto, apenas duas delas (25\%) estavam em tratamento psiquiátrico pouco antes do delito, e igual percentual estava em uso de psicofármacos. Estes achados mostram que as pacientes com comportamento 
homicida da amostra estudada não estavam em tratamento psiquiátrico regular, antes de manifestarem este comportamento. É importante que os serviços de saúde mental trabalhem para prevenir a perda de contato e não-aderência ao tratamento, que frequentemente precedem o comportamento violento cometido por indivíduos com transtornos mentais graves. Também é fundamental que a sociedade e as autoridades governamentais atenuem barreiras de acesso a tratamento psiquiátrico e psicossocial.

Embora as evidências estatísticas e empíricas apontem relação positiva entre transtornos mentais graves e comportamento violento, isso certamente representa pequena proporção da violência ocorrida na comunidade. Em países com altos índices de violência, como o Brasil, onde a violência e a criminalidade têm intensa associação com a precariedade de condições socioeconômicas, o percentual de homicídios associados a transtornos mentais talvez seja ainda menor. O objetivo de pesquisas sobre a associação entre violência e transtornos mentais não é estigmatizar, e, sim, mais bem compreender os fatores que contribuem para esta associação, bem como propor políticas de saúde mental e intervenções terapêuticas para pacientes com transtornos mentais e comportamento violento.

A hipótese de que o transtorno mental tenha efeito especial no impacto da violência da mulher tem sido reforçada por diversos estudos epidemiológicos ${ }^{4}$. Mesmo em estudos em que há diferenças de gênero na violência (apontando os homens como mais violentos), a magnitude destas diferenças parece ser fortemente diminuída quando as amostras consistem de homens e mulheres com transtornos mentais, comparadas a amostras de indivíduos sem estes transtornos ${ }^{25,26}$.

Segundo Hodgins ${ }^{6}$, políticas de saúde mental e práticas clínicas atuais têm falhado em reconhecer que o comportamento agressivo e a vitimização são problemas para muitos pacientes com transtornos mentais graves. Consequentemente, os serviços de atendimento não têm fornecido recursos suficientes para tratar estes problemas, aumentando o número de pacientes transferidos para serviços forenses.

Para Volavka et al.7, o ideal é que estudos futuros sobre comportamento violento na esquizofrenia sejam prospectivos em sua natureza. A variável dependente (violência) deveria ser cuidadosamente definida e a informação sobre ela deveria ser colhida a partir de múltiplos fatores, incluindo auto-relatos, informações de familiares, de serviços de atendimento e de justiça. Os efeitos dos medicamentos antipsicóticos prescritos e a aderência dos pacientes a este tratamento deveriam ser incluídos entre as informações a serem obtidas.

É importante salientar que a conduta criminosa constitui processo sociocultural, visto que os indivíduos com transtornos mentais se inserem neste cenário. Assim, verifica-se que os fatores educacionais e de equilíbrio social contribuem para diminuição de comportamentos de violência na população em geral, assim como entre aqueles com transtornos mentais. O transtorno mental pode funcionar como facilitador de comportamento violento, não como gerador de conduta criminal, por si só.

Tem sido reconhecido que os clínicos subestimam o risco potencial de comportamento violento relacionado às mulheres com psicoses ${ }^{27}$. Consequentemente, este risco pode ser menos considerado, quando o planejamento de manejo deste comportamento é necessário. Com frequência a violência é vista como fenômeno masculino por clínicos e público leigo em geral.

Embora no presente estudo não tenha sido realizada avaliação sobre a frequência de transtornos de personalidade na amostra estudada, uma limitação dele, diversos estudos têm encontrado que a presença destes transtornos aumenta o risco de violência entre indivíduos com transtornos psicóticos ${ }^{28}$. Os transtornos de personalidade também têm sido considerados prevalentes entre mulheres sem psicose que cometem agressão grave ${ }^{29}$.

Outra limitação do presente estudo foi o pequeno tamanho da amostra $(n=8)$. Entretanto, participaram do estudo todas as pacientes com transtornos psicóticos primários que estavam cumprindo medida de segurança em regime de internação hospitalar, por causa de homicídio ou tentativa de homicídio, no estado do Rio de Janeiro. Uma outra limitação foi a utilização de dados retrospectivos, embora todas as pacientes participantes tenham sido entrevistadas durante sua realização. São necessárias novas pesquisas que explorem o risco de violência em mulheres com transtornos mentais de diversas populações e avaliem os benefícios da intervenção terapêutica nestes fatores, na redução do risco de violência.

\section{CONCLUSÃO}

Não obstante a amostra estudada não possa ser considerada representativa de todas as mulheres com transtornos psicóticos que cometeram homicídio, acredita-se que este estudo poderá contribuir para o entendimento e a ilustração da relação entre homicídio e transtornos mentais, nas mulheres. Certamente o estudo de fatores motivadores do comportamento homicida pode fornecer conhecimentos para o estabelecimento de intervenções terapêuticas em mulheres com transtornos mentais que apresentem risco para este ou outros comportamentos violentos.

Pesquisas que forneçam dados que auxiliem a identificação de indivíduos com transtornos mentais com risco de comportamento violento, bem como o tratamento adequado destes indivíduos, podem contribuir para a preven- 
ção deste comportamento, assim como a sua expressão no meio social. Também podem permitir melhor caracterização de grupos ou situações de risco, e esclarecer as motivações específicas relacionadas à manifestação de comportamento violento, em indivíduos com transtornos mentais.

\section{REFERÊNCIAS}

1. Código de Processo Penal. $3^{a}$ ed. São Paulo: Revista dos Tri1. Código de Processo Penal. $3^{a}$ ed. São Paulo: Revista dos Tribunais; 1998.

2. Batista Rosa T, Carvalho MCN. 0 comportamento criminoso: aspectos jurídicos, sociais e psicológicos de homicídio. In: Carvalho MCN, Miranda VR, editores. Psicologia jurídica. Temas de aplicação. Curitiba: Juruá Editora; 2007. p. 159-78.

3. Hodgins S, Mednick SA, Brenann PA, Schulsinger F, Engberg M. Mental disorder and crime. Evidence from a Danish birth cohort. Arch Gen Psychiatry. 1996;53(6):489-96.

4. Swanson JW, Holzer CE, Ganju V, Jono RT. Violence and psychiatric disorder in the community: evidence from the Epidemiologic Catchment Area Surveys. Hosp Community Psychiatry. 1990;41(7):761-70.

5. Moraes T. Início psicopático das esquizofrenias [livre docência]. Rio de Janeiro. Escola de Medicina e Cirurgia. Universidade do Rio de Janeiro; 1970.

6. Hodgins $S$. The major mental disorders and crime: stop debating and start treating and preventing. Int J Law Psychiatry. 2001;24(4-5):427-46.

7. Volavka J, Laska E, Baker S, Meisner M, Czobor P, Krivelevich I. History of violent behavior and schizophrenia in different cultures: analyses based on the WHO study on determinants of outcome of severe mental disorders. Br J Psychiatry. 1997;171:9-14.

8. Teplin I, McClelland GM, Abram KM, Weiner DA. Crime victimization in adults with severe mental ilness: comparison with the National Crime Victimization Survey. Arch Gen Psychiatry. 2005;62:911-21.

9. Modestin J, Hug A, Ammann R. Criminal behavior in males with affective disorders. J Affect Disord. 1997;42(1):29-38.

10. Swartz MS, Swanson JW, Hiday VA, Borum R, Wagner HR, Burns BJ. Violence and severe mental illness: the effects of substance abuse and nonadherence to medication. Am J Psychiatry. 1998;155(2):226-31.

11. Nestor PG. Mental disorder and violence: personality dimensions and clinical features. Am J Psychiatry. 2002;159(12):1973-8.

12. Wallace C, Mullen PE, Burgess P. Criminal offending in schizophrenia over a 25 -year period marked by deinstitutionalization and increasing prevalence of comorbid substance use disorders. Am J Psychiatry. 2004;161(4):716-27.
13. Swanson JW, Swartz MS, Van Dorn RA, Elbogen EB, Wagner HR, Rosenheck RA, et al. A national study of violent behavior in persons with schizophrenia. Arch Gen Psychiatry. 2006:163:490-9.

14. Chaves AC, Shiraawa I. Escalas das síndromes possitiva e negativa e o seu uso no Brasil. In: Goresntein C, Andrade LHSG, Zuardi A, editores. Escalas de avaliação clínica em psiquiatria e psicofarmacologia. São Paulo: Lemos Editorial; 2000.

15. Meehan J, Flynn S, Hunt I, Robinosn J, Bickley H, Parsons R, et al. Perpetrators of homicide with schizophrenia: a national clinical survey in England and Wales. Psychiatr Serv. 2006:57:1648-51.

16. Nielssen $O B$, Westmore $B D$, Large MMB, Hayes RA. Homicide during psychotic illness in New South Wales between 1993 and 2002. Med J Aust. 2007:186:301-4.

17. Link BG, Stueve A. Psychics symptoms and the violent/illegal behavior of mental disorders compared to community controls, in violence and mental disorder: developments and risk assessment. Chicago: University of Chicago Press; 1994.

18. Beck JC. Delusions, substance abuse, and serious violence. J Am Acad Psychiatry Law. 2001;32:169-72

19. Cheung P, Schweitzer I, Crowley K, Tuckwell V. Violence in schizophrenia: role of hallucinations and delusions. Schizophr Res. 1997;26:181-90.

20. American Psychiatric Association. Diagnostic and Statistical Manual of Mental Disorders, 4. ed. Washington, D.C.: American Psychiatric Publishing; 2000.

21. Steinert T. Prediction of inpatient violence. Acta Psychiatr Scand. 2002;106 Suppl 412:133-41.

22. Taylor PJ. Motives for offending among violent and psychotic patients. Br J Psychiatry. 1985;147:491-8

23. Robbins PC, Monahan J, Silver E. Mental disorder, violence and gender. Law Hum Behav. 2003;27:561-71

24. Friedman SH, Hrouda DR, Holden CE, Noffsinger SG, Resnick PJ. Chuld murder committed by severely mentally ill mothers: an examination of mothers found not guilty by reason of insanity. J Forensic Sci. 2005;50:1466-71.

25. Stueve A, Link BG. Gender differences in the relationship between mental illness and violence: evidence from a community-based epidemiological study in Israel. Soc Psychiatry Psychiatr Epidemiol. 1998; 33 Suppl 1:S61-7.

26. Hiday VA, Swartz MS, Swanson JW, Borum R, Wagner HR. Male-female differences in the setting and construction of violence among people with severe mental illness. Soc Psychiatry Psychiatr Epidemiol. 1998;33:568-74

27. McNiel D, Binder R. Clinical assessment of the risk of violence among psychiatric patients. Am J Psychiatry. 1995;152:901-6.

28. Moran P, Walsh E, Tyrer P, Burns T, Creed F, Fahy T. Impact of comorbid personality disorder on violence in psychosis: report from UK700 trial. Br J Psychiatry. 2003;182:129-34.

29. Putkonen H, Komulainen EJ, Virkkunen M, Eronen M, Lonnqvist J. Risk of repeat offending among violent female offenders with psychotic and personality disorders. Am J Psychiatry. 2003;160(5):947-51. 